\title{
Transient perioperative inflammation following lung transplantation and major thoracic surgery with elective extracorporeal support: a prospective observational study
}

\author{
Cecilia Veraar ${ }^{1}$, Stefan Schwarz ${ }^{2}$, Jürgen Thanner ${ }^{2}$, Martin Direder ${ }^{2}$, Panja M. Boehm², \\ Leopold Harnoncourt ${ }^{2}$, Joachim Ortmayr ${ }^{2}$, Clarence Veraar ${ }^{2}$, Julia Mascherbauer ${ }^{3}$, Walter Klepetko ${ }^{2}$, \\ Martin Dworschak ${ }^{1}$, Hendrik J. Ankersmit ${ }^{2,4}$, Bernhard Moser ${ }^{2}$ \\ ${ }^{1}$ Department of Anaesthesiology, General Intensive Care and Pain Medicine, Division of Cardiothoracic and Vascular Anaesthesia and Intensive \\ Care Medicine, Medical University of Vienna, Vienna, Austria; ${ }^{2}$ Department of Surgery, Division of Thoracic Surgery, Medical University of Vienna, \\ Vienna, Austria; ${ }^{3}$ Department of Internal Medicine II, Division of Cardiology, Medical University of Vienna, Vienna, Austria; ${ }^{4}$ Head FFG Project \\ “APOSEC", FOLAB Surgery, Medical University Vienna, Vienna, Austria \\ Contributions: (I) Conception and design: C Veraar, HJ Ankersmit, B Moser; (II) Administrative support: C Veraar, HJ Ankersmit, B Moser; (III) \\ Provision of study materials or patients: W Klepetko, HJ Ankersmit, B Moser; (IV) Collection and assembly of data: S Schwarz, J Thanner, M \\ Direder, PM Boehm, L Harnoncourt, J Ortmayr, C Veraar; (V) Data analysis and interpretation: J Mascherbauer, W Klepetko, M Dworschak, HJ \\ Ankersmit, B Moser; (VI) Manuscript writing: All authors; (VII) Final approval of manuscript: All authors. \\ Correspondence to: Bernhard Moser, MD, PD, Assoc. Prof., MBA, FEBTS. Department of Surgery, Division of Thoracic Surgery, Medical University \\ of Vienna. Waehringer Guertel 18-20. 1090 Vienna, Austria. Email: bernhard.moser@meduniwien.ac.at.
}

Background: The clinical relevance of inflammation induced by elective perioperative extracorporeal membrane oxygenation (ECMO) usage as an integral part of modern lung transplantation (LUTX) remains elusive. The aim of this study was to determine the perioperative cytokine response accompanying major thoracic surgery employing different extracorporeal devices comprising ECMO, cardiopulmonary bypass $(\mathrm{CPB})$, or no extracorporeal circulation in relation to inflammation, clinically tangible as increased sequential organ failure assessment (SOFA) score, called SOFA.

Methods: In this prospective, observational pilot study 42 consecutive patients with end-stage pulmonary disease undergoing LUTX; 15 patients with chronic thromboembolic pulmonary hypertension (CTEPH) undergoing pulmonary endarterectomy and 15 patients with lung cancer undergoing major lung resections were analysed. Cytokine serum concentrations and SOFA were determined before, at end of surgery and in the following postoperative days.

Results: LUTX on ECMO and pulmonary endarterectomy (PEA) on CPB triggered an immediate increase in cytokine serum concentrations at end of surgery: IL-6: 66-fold and 71-fold, IL-10: 3-fold and 2.5-fold, ST2/IL-33R: 5-fold and 4-fold and SOFA: 10.5 \pm 2.8 and 10.7 \pm 1.7 , that decreased sharply to baseline levels from postoperative day $1-5$. Despite low perioperative mortality (3 patients, $4.1 \%$ ) extremely high SOFA $\geq 13$ was associated with mortality after LUTX. Delta-SOFA distinguished survivors from nonsurvivors: $-4.5 \pm 3.2$ vs. $-0.3 \pm 1.5$ ( $\mathrm{P}=0.001)$. Increased IL-6 serum concentrations were predictive for increased SOFA (sensitivity: 97\%, specificity: 80\%). Peak cytokine serum concentrations correlated with ECC duration, maximal lactate, transfusion of red-blood-cells, fresh-frozen-plasma, and catecholamine support.

Conclusions: LUTX and PEA on extracorporeal circulation with an excellent outcome triggered an immediate rise and concomitant fall of inflammation as observed in cytokine serum concentrations and SOFA. High absolute SOFA in the presence of an uncomplicated postoperative course may pertain to specific management strategies rather than organ failure.

Keywords: Extracorporeal membrane oxygenation (ECMO); lung transplantation (LUTX); pulmonary endarterectomy (PEA); perioperative inflammation; sequential organ failure assessment (SOFA) 
Submitted Jun 17, 2020. Accepted for publication Nov 27, 2020.

doi: $10.21037 /$ atm-20-4771

View this article at: http://dx.doi.org/10.21037/atm-20-4771

\section{Introduction}

Over the years, the indications for deployment of extracorporeal membrane oxygenation (ECMO) have expanded from resuscitation of patients with acute respiratory and cardiac failure to elective and semi-elective thoracic surgical procedures. ECMO is a technology capable of providing short- and long-term mechanical support to the heart, lungs, or both (1). At our institution the first case series report on the successful use of ECMO for the treatment of graft failure after cardiac transplantation-when weaning from cardiopulmonary bypass(CPB) was not possible-was published in 2001 (2). In the same decade, ECMO gradually evolved as a safe intraoperative support alternative to $\mathrm{CPB}$, enabling the resection of locally advanced intrathoracic malignancies and complex tracheobronchial procedures (3). Subsequently, the use of veno-arterial (v/a) ECMO support was integrated in standard surgical treatment strategies for patients undergoing lung transplantation (LUTX) and for postoperative hemodynamic and respiratory stability in selected patients with poor initial graft function (prolonged $\mathrm{ECMO}$ ). Veno-venous (v/v) ECMO is increasingly employed to bridge patients with respiratory failure to LUTX (4-6).

Previous investigations have shown that the use of $\mathrm{CPB}$ induces a brief pro-inflammatory response, clinically determined by the sequential organ failure assessment (SOFA) followed by a long-lasting second phase of immune suppression (7). The SOFA score was designed to clinically measure organ dysfunction by assessing the respiratory, coagulation, hepatic, cardiovascular, renal and neurological function in patients admitted to the ICU (8). The exposure of a patient's blood to foreign surfaces of the CPB circuit is known to imbalance the inflammatory system via blood flow shear stress, expression of cytokines, activation of the complement system and dysfunctions of the coagulation system $(1,9)$. This broad wave of systemic inflammation has been linked to adverse clinical outcomes ranging from mild adverse effects such as fever or diffuse tissue edema, to moderate adverse effects comprising pathological hemodynamic instability or coagulopathy, to severe complications including acute organ injury and even mortality $(10,11)$.

A similar response induced by ECMO was observed in patients with acute refractory cardiac and respiratory failure requiring immediate life-saving circulatory and respiratory support (12). Experimental and animal studies have shown that already two hours after initiation of ECMO support, pro- and anti-inflammatory cytokines [interleukin (IL)$1 \beta$, IL-6, IL-10 and tumour necrosis factor (TNF)- $\alpha$ ] were significantly up-regulated $(13,14)$. Several studies investigated the inflammatory response to ECMO during resuscitation, whereas the use of elective intraoperative and prolonged ECMO support has never been systematically investigated (15).

The main objective of this study with observational design was to investigate the perioperative inflammatory response of thoracic surgical procedures employing extracorporeal circulation (ECC) comprising LUTX on ECMO, pulmonary endarterectomy (PEA) on CPB; and patients undergoing pulmonary resections without ECC. Sub-analysis was performed among LUTX patients with different underlying diagnoses [chronic obstructive pulmonary disease (COPD), cystic fibrosis (CF), idiopathic pulmonary fibrosis (IPF) and idiopathic pulmonary arterial hypertension (IPAH)]. The perioperative inflammatory response was elicited by cytokine measurements and SOFA. We attempted to identify predisposing factors of clinical(SOFA) and experimental origin(cytokines) for the early detection of worse clinical outcome after major thoracic surgery, employing elective ECMO or CPB. We present the following article in accordance with the STROBE reporting checklist (available at http://dx.doi. org/10.21037/atm-20-4771).

\section{Methods}

The study was conducted in accordance with the Declaration of Helsinki (as revised in 2013). The study was approved by the Institutional Ethics Committee of the Medical University of Vienna (EK1363/2018). Written informed consent was obtained from all study participants.

\section{Study design, setting and patients}

The study was designed as an explorative prospective pilot and cohort study combining clinical and experimental 
research. It was designed as a purely observational study and performed at the Medical University of Vienna.

We included 42 consecutive patients with end-stage pulmonary disease [COPD $(\mathrm{n}=15), \mathrm{CF}(\mathrm{n}=15), 123 \mathrm{IPF}$ $(\mathrm{n}=7)$, IPAH $(\mathrm{n}=5)$ ] undergoing LUTX on ECMO; 15 consecutive chronic thromboembolic pulmonary hypertension (CTEPH) patients undergoing PEA on $\mathrm{CPB}$ and 15 consecutive lung cancer patients undergoing major lung resections without ECC during a period of 12 months, from May 2018 until April 2019. We excluded pregnant women, patients who were younger than 18 years and patients who did not give written informed consent. Blood samples were drawn before surgery, at ICU admission, once on each of the 3 following post- operative days (PODs) and at POD5. IL-6, IL-10, ST2/IL-33R, TNF- $\alpha$ and transforming growth factor (TGF) $\beta$ serum concentrations were measured by commercially available enzyme-linked immunosorbent assay (ELISA) kits.

\section{Sandwich ELISA technique}

IL-6, IL-10, ST2/IL-33R, TNF- $\alpha$ and Transforming Growth Factor (TGF)- $\beta$ serum concentrations were measured by commercially available enzyme-linked immunosorbent assay (ELISA) kits (R\&D Systems, Minneapolis, Minnesota, United 130States) according to the manufacturers' instructions. 96-well microplates were incubated with capture antibodies (Mouse anti-human IL-6, IL-10, ST2, TNF- $\alpha$ and TGF- $\beta$ ) overnight at room temperature. Blocking was done with assay buffer. After incubation with serum samples and washing, HRP-conjugated detection antibodies were added (Biotinylated goat anti-human IL-6, IL-10, ST2, TNF- $\alpha$ and TGF- $\beta$ ). A color reaction was obtained with peroxidase reagent tetramethylbenzidine (TMB) (Sigma-Aldrich Corp., St. Louis, MO, USA) and the optical density (OD) was read at $450 \mathrm{~nm}$ using an absorbance microplate reader for ELISA, the Infinite F50 (Tecan, Männedorf, Switzerland).

\section{Perioperative management}

\section{Anesthesia}

All patients enrolled received standard perioperative monitoring after entering the operating room. Anesthesia was induced with $2 \mathrm{mg}$ Midazolam, Fentanyl $0.2 \mu \mathrm{g} / \mathrm{kg}$, Propofol $2 \mathrm{mg} / \mathrm{kg}$ and Cis-Atracurium $0.2 \mathrm{mg} / \mathrm{kg}$ (PEA and LUTX)/Rocuronium $0.5 \mathrm{mg}$ (lung resection). Antibiotic prophylaxis was administered: piperacillin/tazobactam $4.5 \mathrm{~g}$ for PEA and LUTX and cefuroxime for lung resection within
30 to 60 minutes before incision. A Swan-Ganz catheter and a central venous catheter were placed into the right or left jugular vein to measure pulmonary artery pressures in patients undergoing LUTX and PEA. Anesthesia was maintained with fentanyl via perfusion or bolus and propofol $6 \mathrm{mg} / \mathrm{kg} / \mathrm{h}$ for PEA and LUTX and sevoflurane 1 minimal alveolar concentration for lung resection. Transesophageal echocardiography was installed in patients undergoing PEA or LUTX to monitor cardiac function, volume status and adrenergic support during surgery. While an initial dose of $60 \mathrm{IU} / \mathrm{kg}$ of heparin was administered before initiation of intraoperative veno-arterial (v/a) ECMO for patients undergoing LUTX, $400 \mathrm{IU} / \mathrm{kg}$ of heparin were given before introduction of $\mathrm{CPB}$ for patients undergoing PEA. Normothermic conditions of $37^{\circ} \mathrm{C}$ were pursued for patients undergoing LUTX and lung resection. Patients undergoing PEA were cooled to $18{ }^{\circ} \mathrm{C}$ (deep hypothermia) to endure periods of complete circulatory arrest.

\section{Surgery}

Donor lungs were harvested during multi-organ procurement preserved with colloid containing low potassium solution and kept inflated during transport. LUTX was performed through bilateral thoracotomy or clamshell incision. LUTX was performed with intraoperative central v/a ECMO with and without prolongation into the postoperative period. In patients with prolonged v/a ECMO, central cannulation was switched to the peripheral location in the groin after implantation of the lungs (16).

PEA was performed through a median sternotomy requiring $\mathrm{CPB}$ with bicaval cannulation. After aortic cross-clamping, cardioplegia was administered and deep hypothermic circulatory arrest was employed to improve visualization of the pulmonary arteries. Bilateral PEA was performed sequentially in all patients (17).

Lung resections were performed via muscle-sparing antero-lateral thoracotomies. Only patients undergoing anatomic pulmonary resection (lobectomy/pneumonectomy) during single lung ventilation (double-lumen intubation) were included in this study.

\section{Extracorporeal circulation (ECC)}

Bridging strategies were chosen according to patient's hemodynamic and respiratory conditions. Patients with severe hypoxic respiratory failure received a v/v ECMO (Cardiohelp, Oygenator Quadrox)/(Xenios, Oxygenator Hilite) with a 2 -site (femoro-jugular) or single-site large- 
bore double-lumen cannula ranging from $27 \mathrm{~F}$ to $31 \mathrm{~F}$ (Avalon Laboratories, Los Angeles, Calif) (18).

All patients were transplanted on intraoperative heparinbound v/a ECMO (Medtronic Carmeda), hollow-fiber oxygenator (Medtronic), centrifugal pump (Biomedicus), flow probes and 3/8- inch internal diameter (18).

PEA for CTEPH patients was performed with CPB. The $\mathrm{CPB}$ circuit was primed $(1,000 \mathrm{~mL}$ crystalloid and $500 \mathrm{~mL}$ colloid solution together with 5,000 IE heparin, and $100 \mathrm{~mL}$ mannitol 20\%) according to institutional standards. CPB was performed using non-pulsatile flow at $2.5 \mathrm{~L} / \mathrm{min} / \mathrm{m}^{2}$, a non-heparin-coated circuit, and a membrane oxygenator (Quadrox $^{\mathrm{TM}}$, Maquet, Hirrlingen, Germany, or Capiox, Terumo, Eschborn, Germany) (19).

For prolonged ECMO support a femoro-femoral v/a ECMO using $17 \mathrm{~F}$ to $19 \mathrm{~F}$ drainage and $15 \mathrm{~F}$ to $17 \mathrm{~F}$ reperfusion cannulas was inserted (all Bio-Medicus Cannula, Medtronic Inc, St Paul, Minneapolis). Anticoagulation during prolonged ECMO support was managed by subcutaneous administration of low-molecular-weight heparin. ECMO was continued until patients were hemodynamically stable, had normal chest X-ray, adequate oxygenation (fraction of inspired oxygen $<0.5$ ), low ventilation pattern and had a normalized fluid balance.

\section{Postoperative management}

Patients undergoing LUTX received induction therapy with alemtuzumab in $84 \%$ or Anti-thymocyte globulin in $3 \%$ or no induction therapy (13\%) directly after ICU admission. Maintenance therapy was employed in all patients with tacrolimus, mycophenolat-mofetil (only in the non-alemtuzumab group) and corticosteroids. All patients undergoing LUTX received postoperative antiinfectious prophylaxis therapy with piperazillin/tazobactam, a lifelong Pneumocystis prophylaxis with trimethoprimsulfamethoxazole, prophylactic inhalation therapy with amphotericin $\mathrm{B}$ and gentamicin and CMV prophylaxis including CMV hyperimmuno-globulines (POD 1, 7, 14 and 21) together with valganciclovir. Patients undergoing PEA received piperazillin/tazobactam or cefazolin for postoperative antibiotic prophylaxis. Patients undergoing lung resection did not routinely receive postoperative antibiotic therapy.

\section{Definition of SOFA}

SOFA was assessed every $24 \mathrm{~h}$ for all patients admitted to the ICU (LUTX and PEA patients). The SOFA score was calculated by assessing each of the 5 organ dysfunctions (respiratory, coagulation, hepatic, cardiovascular and renal) from 0-4 points. The Assessment of the neurological function by using the Glasgow coma scale (GCS) was excluded due to limited evaluability in actively sedated patients. The following data was collected: need for mechanical ventilation, arterial partial pressure of oxygen to fraction of inspired oxygen $\left(\mathrm{PaO}_{2} / \mathrm{FiO}_{2}\right)$ ratio, platelet count, bilirubin, mean arterial pressure, doses of adrenergic agents, creatinine, and urine output (7).

\section{Statistical analysis}

Normally distributed data were reported as mean \pm SD, nonnormal distributions as median (range). Mann-Whitney $\mathrm{U}$ test was employed for non-normally distributed data and $t$-tests for parametric data. Pearson's correlation-coefficient $r$ was employed to measure the strength of the linear relationship between cytokine concentrations and clinical data. We plotted the receiver operating characteristic (ROC) curve, calculated the Youden Index to identify optimal cut-offs for cytokine concentrations at the end of surgery. We performed binary logistic regression to evaluate the negative predictive value (NPV), positive predictive value (PPV), sensitivity, specificity, and the OR to predict postoperative SOFA ( $>10$ points). Chisquare tests were employed to test relationships between categorical variables. Kaplan Mayer analysis was performed for survival analysis. The level of statistical significance was set at 0.05 (two-tailed $\mathrm{P}$ values). Statistical analyses and visualization were performed using SPSS software (version 25; IBM SPSS Inc., IL, USA) and GraphPad Prism 5 (GraphPad Software, La Jolla, CA, USA). Boxplots were designed as followed: Box: 1st to 3rd quartile, Bar: median, Whiskers: percentile 5-95, Outliers: all shown as dots.

\section{Results}

Demographic data and perioperative characteristics are depicted in Table 1. Five patients $(11 \%)$ underwent ReLUTX due to chronic lung allograft dysfunction. Among these patients three were initially diagnosed with $\mathrm{CF}$, one had IPF and one was initially suffering from IPAH.

Nine percent of all patients undergoing LUTX (COPD, $\mathrm{CF}$, IPF, and IPAH) were bridged to transplantation via semi-elective v/v ECMO, 100\% received intraoperative, elective v/a ECMO and 19\% received prolonged semielective v/a ECMO after surgery, respectively. All patients 
Table 1 Basic demographic, procedural and outcome data

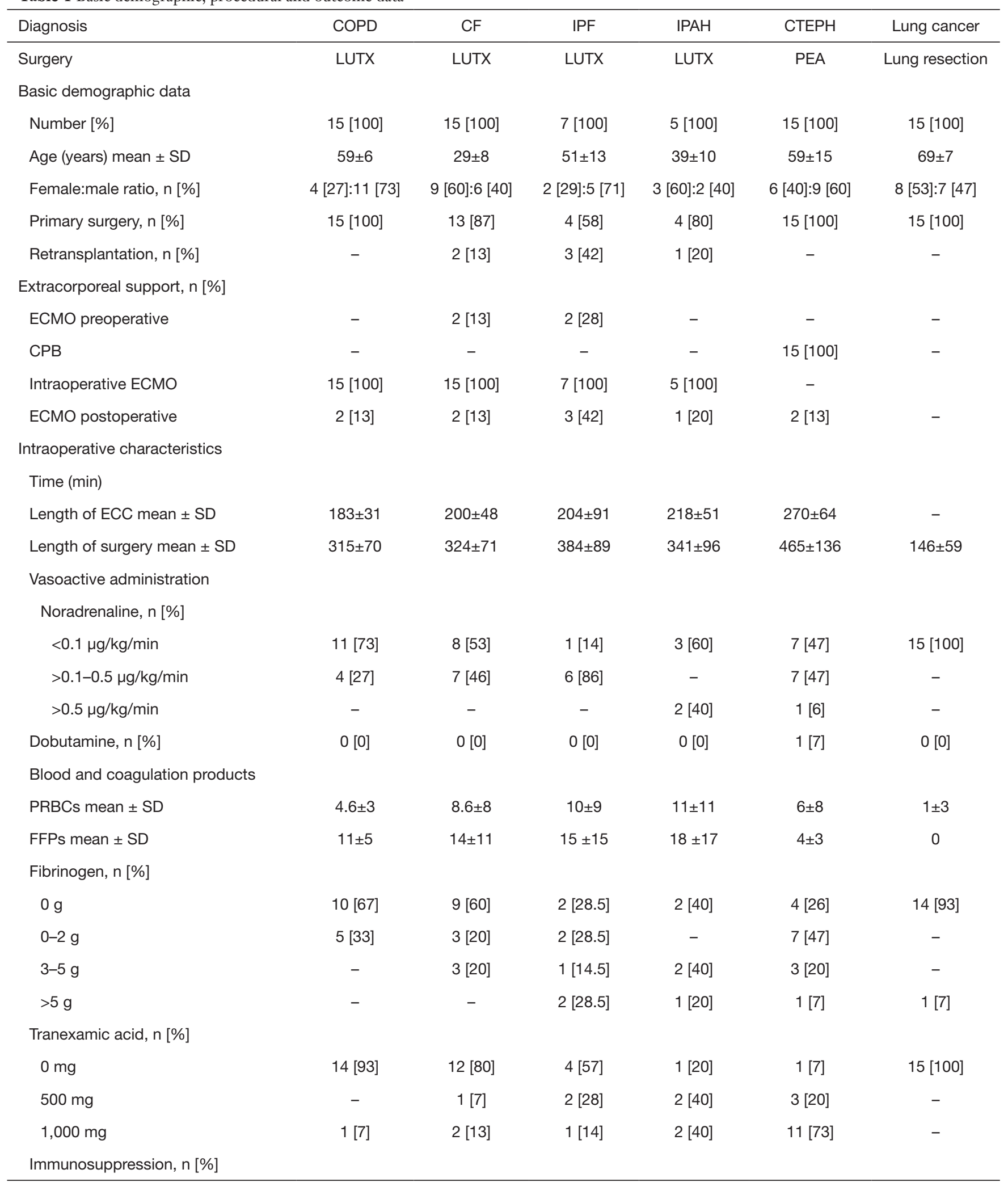

Table 1 (continued) 
Table 1 (continued)

\begin{tabular}{|c|c|c|c|c|c|c|}
\hline Diagnosis & COPD & CF & IPF & IPAH & CTEPH & Lung cancer \\
\hline Hydrocortisone $100 \mathrm{mg}$ & - & - & - & - & 15 [100] & - \\
\hline \multicolumn{7}{|c|}{ Measurement of serum parameters } \\
\hline Max. $\mathrm{BL}(\mathrm{mg} / \mathrm{dL})$ mean $\pm \mathrm{SD}$ & $3.1 \pm 1.2$ & $3.5 \pm 1.1$ & $4.2 \pm 1.4$ & $4.0 \pm 1.8$ & $4.3 \pm 0.8$ & $1.5 \pm 0.7$ \\
\hline \multicolumn{7}{|c|}{ Postoperative characteristics (first 24 h) } \\
\hline SOFA-Score mean \pm SD & $9.2 \pm 1.4$ & $10.9 \pm 3$ & $11.2 \pm 4.6$ & $11.8 \pm 1.3$ & $10.72 \pm 1.7$ & - \\
\hline qSOFA-Score mean \pm SD & - & - & - & - & - & $0[0]$ \\
\hline SBP $<100$ & - & - & - & - & - & $0[0]$ \\
\hline \multicolumn{7}{|l|}{ Outcome analysis, n [\%] } \\
\hline Revision, mean \pm SD & $1[7]$ & $0[0]$ & $0[0]$ & $0[0]$ & $0[0]$ & $2[13]$ \\
\hline $\mathrm{VAC}$, mean $\pm \mathrm{SD}$ & $1[7]$ & $0[0]$ & $0[0]$ & $0[0]$ & $0[0]$ & $0[0]$ \\
\hline $\mathrm{POS}$, mean $\pm \mathrm{SD}$ & $2[13]$ & $0[0]$ & $2[25]$ & $2[40]$ & $2[13]$ & - \\
\hline 30-d mortality, mean \pm SD & $1[7]$ & $0[0]$ & $1[12]$ & $0[0]$ & $1[7]$ & $0[0]$ \\
\hline $\mathrm{HF}$, mean $\pm \mathrm{SD}$ & $1[7]$ & $1[7]$ & $1[7]$ & $2[40]$ & $2[13]$ & $0[0]$ \\
\hline
\end{tabular}

$\mathrm{BL}$, blood lactate concentration; bpm, beats per minute; CF, cystic fibrosis; COPD, chronic obstructive pulmonary disease; CPB, cardiopulmonary bypass; d, day; ECC, extracorporeal circulation; ECMO, extracorporeal membrane oxygenation; FFP, fresh frozen plasma; HB, haemoglobin; G/I, Giga per liter; HR heart rate; IPAH, idiopathic pulmonary hypertension IPF idiopathic pulmonary fibrosis; LUTX, lung transplantation; n, number; PEA, pulmonary endarterectomy; POS, psycho-organic syndrome; PRBCs, packed red blood cells; $\mathrm{RR}$, respiratory rate; SBP, systolic blood pressure; SD, standard deviation; SOFA, sequential organ failure assessment score; VAC, vacuum assisted closure-therapy; WBC, white blood cells.

undergoing PEA (CTEPH) had CPB during surgery and $13 \%$ required postoperatively prolonged semi-elective v/a ECMO support. Patients undergoing lung resection for cancer did not require ECC support. The peak SOFA was at $10.5 \pm 2.8$ for LUTX on ECMO and $10.7 \pm 1.7$ for PEA on $\mathrm{CPB}$ patients at end of surgery and decreased steadily thereafter.

\section{Treatment differences between study groups}

In patients undergoing LUTX on ECMO support there was no difference among the different underlying diagnoses (COPD, CF, IPF, and IPAH) with respect to the number of administered units of packed red blood cells (PRBC) $(\mathrm{P}=0.119)$, units of fresh frozen plasma (FFP) $(\mathrm{P}=0.623)$, grams of fibrinogen $(\mathrm{P}=0.083)$, dosage of noradrenalin $(\mathrm{P}=0.107)$, blood lactate concentration $(\mathrm{P}=0.311)$, haemoglobin concentration $(\mathrm{P}=0.841)$, ECCtime $(\mathrm{P}=0.526)$ and time of surgery $(\mathrm{P}=0.187)$. In contrast there are inherent treatment differences comparing patients undergoing LUTX on ECMO and patients undergoing PEA supported by CPB: units of PRBC $(\mathrm{P}=0.026)$, units of FFP $(\mathrm{P}<0.001)$, blood lactate concentration $(\mathrm{P}=0.021)$, ECC time $(\mathrm{P}<0.001)$ and time of surgery $(\mathrm{P}<0.001)$, but no difference in the administration of grams of fibrinogen $(\mathrm{P}=0.156)$ and dosage of noradrenalin $(\mathrm{P}=0.940$, Table 1).

\section{Increased postoperative cytokine release}

Baseline preoperative cytokine concentrations did not reveal statistically significant differences between patients planned for LUTX on ECMO, PEA on CPB or pulmonary 


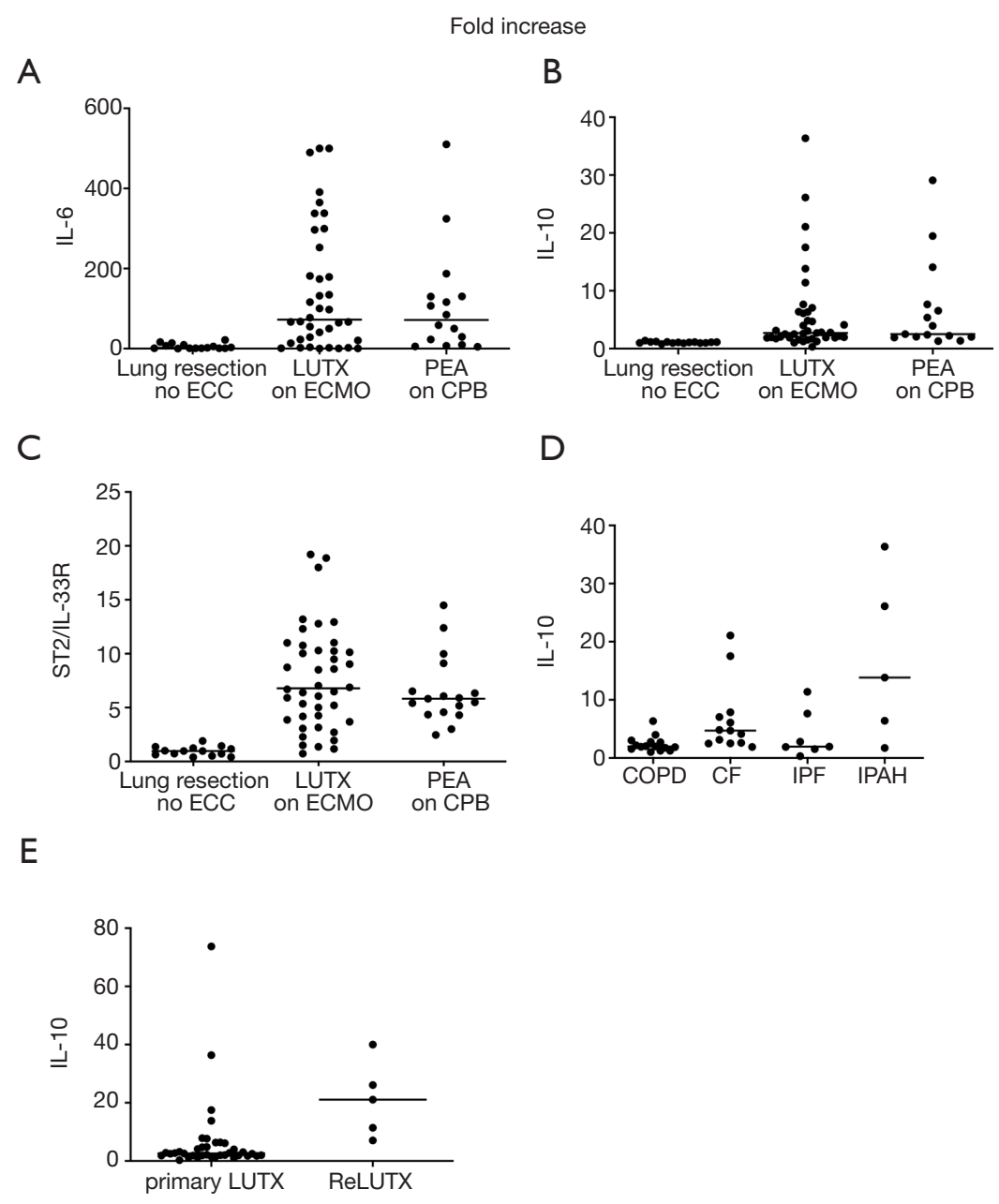

Figure 1 Increased cytokine expression after LUTX and PEA were depicted as fold increase (increase from baseline prior to surgery to ICU admission). Serum cytokine expressions including IL-6 (A), IL-10 (B) and ST2/IL33 (C) of patients undergoing LUTX with ECMO and PEA with CPB; and patients undergoing lung resection without ECC were depicted. A statistically significant increase in IL- 10 serum concentrations among patients undergoing LUTX with CF and IPAH compared to COPD patients from baseline prior to surgery to peak concentrations after surgery were shown in (D). Significantly increased IL-10 serum concentrations from baseline to end of surgery in patients undergoing primary LUTX compared to patients undergoing Re-LUTX are presented in (E). LUTX, lung transplantation; PEA, pulmonary endarterectomy; ECMO, extracorporeal membrane oxygenation; ECC, extracorporeal circulation; COPD, chronic obstructive pulmonary disease; LUTX, lung transplantation.

resections without extracorporeal support.

IL-6 serum concentrations increased 66-fold after LUTX on ECMO support, 71-fold after PEA on CPB and 2-fold in patients undergoing lung resection without $\mathrm{ECC}$ (Figure 1A).

Patients undergoing LUTX on ECMO had an 8-fold, PEA on $\mathrm{CPB}$ a 7 -fold and patients undergoing pulmonary resections without ECC had no increase of IL-10 serum concentrations, respectively (Figure $1 B$ ).
ST2/IL-33R serum concentrations increased 5-fold in patients who underwent LUTX on ECMO, 4-fold in patients after PEA on CPB, but not in patients with pulmonary resections without ECC (Figure 1C).

TNF- $\alpha$ serum concentrations increased 15 -fold in LUTX on ECMO, 3-fold in PEA on CPB but not in pulmonary resections without ECC. There were no alterations in TGF- $\beta$ serum concentrations. 


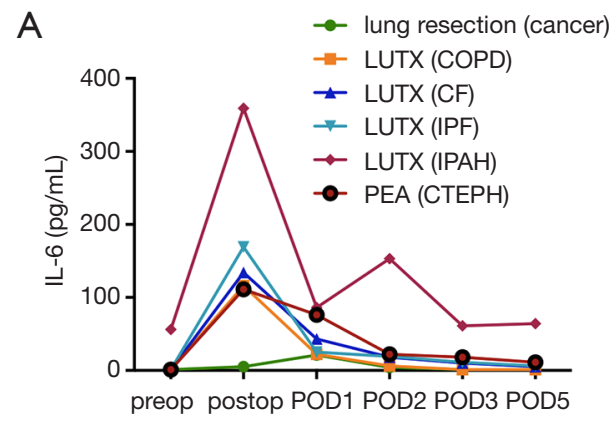

C

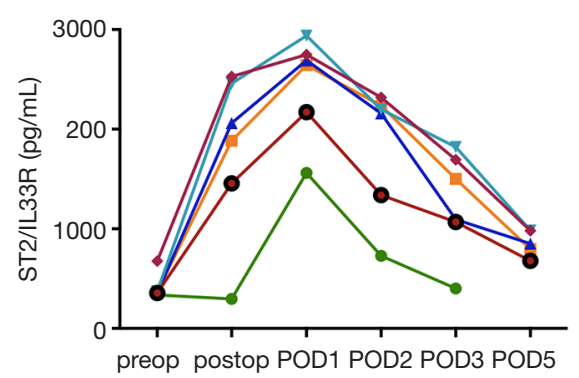

B

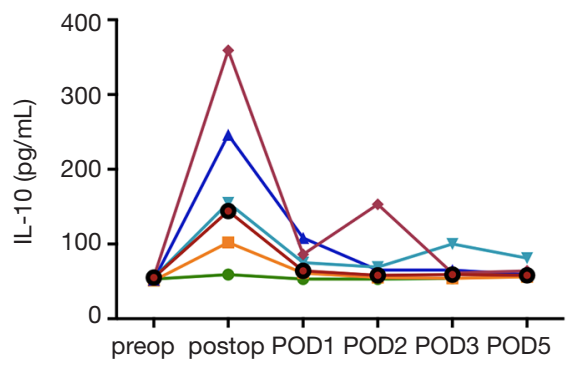

D

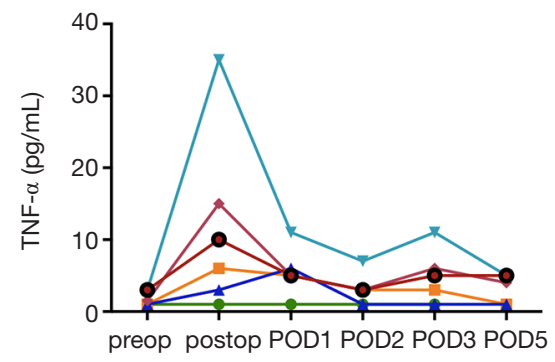

Figure 2 Dynamic cytokine-expression in the perioperative period. The dynamic perioperative cytokine release is shown in IL-6 (A), IL-10 (B), ST2/IL33 (C) and TNF- $\alpha$ (D).

The underlying diagnoses influence IL-10 serum concentrations in patients undergoing LUTX (COPD, CF, IPF, IPAH)

IL-10 serum concentrations increased significantly from baseline prior to surgery to peak concentrations after surgery between patients undergoing LUTX for COPD [51 (range, 49-69) to 102 (range, 62-321) pg/mL] and CF [52 (range, $48-88)$ to 246 (range, $125-6,514) \mathrm{pg} / \mathrm{mL}](\mathrm{P}<0.001)$ or IPAH [56 (range, 50-76) to 705 (range, 100-2,104) pg/mL] $(\mathrm{P}<0.019)$ (Figure 1D).

\section{Increased preoperative IL-10 concentrations in patients bridged to LUTX}

Only preoperative IL-10 concentrations were significantly increased in patients with implanted v/v ECMO: [55 (range, 54-908) $\mathrm{pg} / \mathrm{mL}$ ] compared to no ECMO bridging: [53 (range, 42-104) pg/mL] $(\mathrm{P}=0.045$, Figure 1E).

\section{Dynamic changes of cytokine-expression after surgery}

IL-6 serum concentrations increased significantly from baseline to end of surgery and decreased significantly at POD1 in all patients. From POD1 to POD2 there was a significant decrease in IL-6 concentrations in patients with LUTX (COPD, CF) and PEA (CTEPH) and a second significant increase in patients with LUTX (IPAH) (Figure 2A). IL-6 serum concentrations increased 116-fold in COPD, 28-fold in CF, 31-fold in IPF, 36-fold in IPAH, 71-fold in CTEPH and 2-fold in patients with lung resection from baseline to end of surgery.

IL-10 serum concentrations increased in LUTX (COPD, $\mathrm{CF}, \mathrm{IPAH})$ and PEA (CTEPH) patients form baseline to end of surgery; and decreased statistically significant in the following postoperative days (Figure 2B). IL-10 serum concentrations increased 2-fold in COPD, 5-fold in CF, 2-fold in IPF, 13-fold in IPAH, 2.5-fold in CTEPH and 1 -fold in patients with lung resection from baseline to end of surgery.

Peak ST2/IL-33R concentrations were detected at POD1 in all study groups followed by a decrease starting on POD2. ST2/IL-33R levels rose significantly in patients with LUTX (COPD, CF) and PEA (CTEPH) from baseline to end of surgery, from end of surgery to POD1 and decreased significantly at all consecutive time points. Patients with LUTX (IPAH) had only a significant increase from baseline to end of surgery and a significant decrease 
from POD2 to POD3 and POD5. The lung resection group rose significantly from end of surgery to POD1 and decreased at all following time-points (Figure 2C). ST2/IL$33 \mathrm{R}$ serum concentrations increased 4-fold in COPD, 5-fold in CF, 5 -fold in IPF, 3-fold in IPAH, 4-fold in CTEPH and 1 -fold in patients with lung resection from baseline to end of surgery.

TNF- $\alpha$ serum concentrations rose significantly in LUTX (COPD, IPF) and PEA (CTEPH) patients from baseline to end of surgery. A significant decrease from end of surgery to POD1 was only observed in patients with PEA (CTEPH) (Figure 2D). TNF- $\alpha$ serum concentrations increased 6-fold in COPD, 1-fold in CF, 7-fold in IPF, 3-fold in IPAH, 2.5fold in CTEPH and 1-fold in patients with lung resection from baseline to end of surgery.

There were no alterations in TGF- $\beta$ serum concentrations in all time-points.

\section{Correlation between cytokine expression and clinical parameters}

Correlations among clinical parameters and fold-increase of cytokine concentrations from baseline prior to surgery to postoperative peak serum concentrations are depicted in Table 2. A significant correlation among IL-10, ST2/ IL33R, CRP serum concentrations and ECC length could be observed. Total operation time did not show any impact on serum cytokine concentrations. High maximum lactate concentrations were significantly associated with high foldincrease of IL-10, TGF- $\beta$ and CRP levels. Packed red cells counts, fresh frozen plasmas (FFPs) and dosages of noradrenaline correlated significantly with IL-10 and IL-6 fold-increases. Low haemoglobin levels were only associated with statistically significant IL-6 fold-increases.

There was no association between cytokine serum concentrations and primary graft dysfunction (PGD) grading (supplement).

\section{Cytokines are prognostic of inflammation as defined by SOFA}

The sensitivity, specificity, PPV, NPV and OR of all cytokines for predicting increased perioperative inflammation as defined by SOFA criteria are detailed in Table 3. The following Youden indices divided patients into groups of low and high serum concentrations of the respective cytokines for SOFA >10: IL-10 $311 \mathrm{ng} / \mathrm{mL}$, IL-6 $69.0 \mathrm{ng} / \mathrm{mL}$, TNF- $\alpha 2.3 \mathrm{ng} / \mathrm{mL}$, ST2/IL33R 2,159 ng/mL,
TGF- $\beta 17.2 \mathrm{ng} / \mathrm{mL}$. IL-6 serum concentrations at end of surgery had the highest OR 18.6 and the highest sensitivity of $97 \%$ for SOFA, respectively.

\section{SOFA and relative SOFA changes $(\triangle-S O F A)$ are associated with postoperative outcome}

Kaplan Mayer survival analysis (cut-off SOFA $\geq 13$ ) revealed a significantly greater survival for patients with SOFA $<13$ $(\mathrm{P}=0.02)$.

Focusing on patients who underwent LUTX a change in SOFA score $(\Delta$-SOFA) from end of surgery to POD3 was at $-3.9 \pm 3.3$ for COPD, $-5.5 \pm 2.5$ for $\mathrm{CF},-3 \pm 4.9$ for IPF, and $-5.2 \pm 2.3$ for IPAH. Patients who underwent PEA for CTEPH displayed a $\triangle$-SOFA of $-4.7 \pm 3.5$ (Figure $3 A$ ). $\Delta$-SOFA decreased at $-1.5 \pm 4.9$ for re-intubated and $-4.6 \pm 3.3$ for patients who remained extubated $(\mathrm{P}<0.001)$. Patients requiring hemofiltration compared to those without had a $\Delta$-SOFA of $-1.2 \pm 1.4 v s .-5.1 \pm 3.2(\mathrm{P}<0.001)$ (Figure $3 B$ ). $\Delta$-SOFA of patients who deceased in the early postoperative period was at $-0.3 \pm 1.5$ vs. $-4.8 \pm 3.2(\mathrm{P}<0.001)$ in patients who survived, respectively (Figure 3C).

\section{Surgical revisions}

Three patients underwent surgical revision one patient after LUTX on ECMO and two patients after lung resection. One CF patient who underwent LUTX newly developed a partial anastomotic dehiscence on POD14 (diagnosed via newly developed fluid pneumothorax on chest X-ray followed by bronchoscopy) that was successfully corrected via right upper lobe lobectomy and reanastomosis of the intermediate bronchus to the right main bronchus. One patient with advanced pulmonary emphysema who underwent lobectomy of the right lower lobe plus decortication of the upper lobe for lung cancer in conjunction with recurrent pleuritis was surgically revised for persistent air leak on POD8. Another patient who underwent lobectomy of the left upper lobe together with anatomic segmentectomy of the segment 6 developed pneumonia in the remaining left lower lobe on POD5 and was surgically revised for narrowing at the site of the left upper lobe bronchial stump via a bronchial sleeve resection.

\section{Mortality}

Perioperative (30-day) mortality of the entire study patient cohort was $4.1 \%$. One patient bridged with v/v ECMO 
Table 2 Correlation of cytokines with clinical parameters

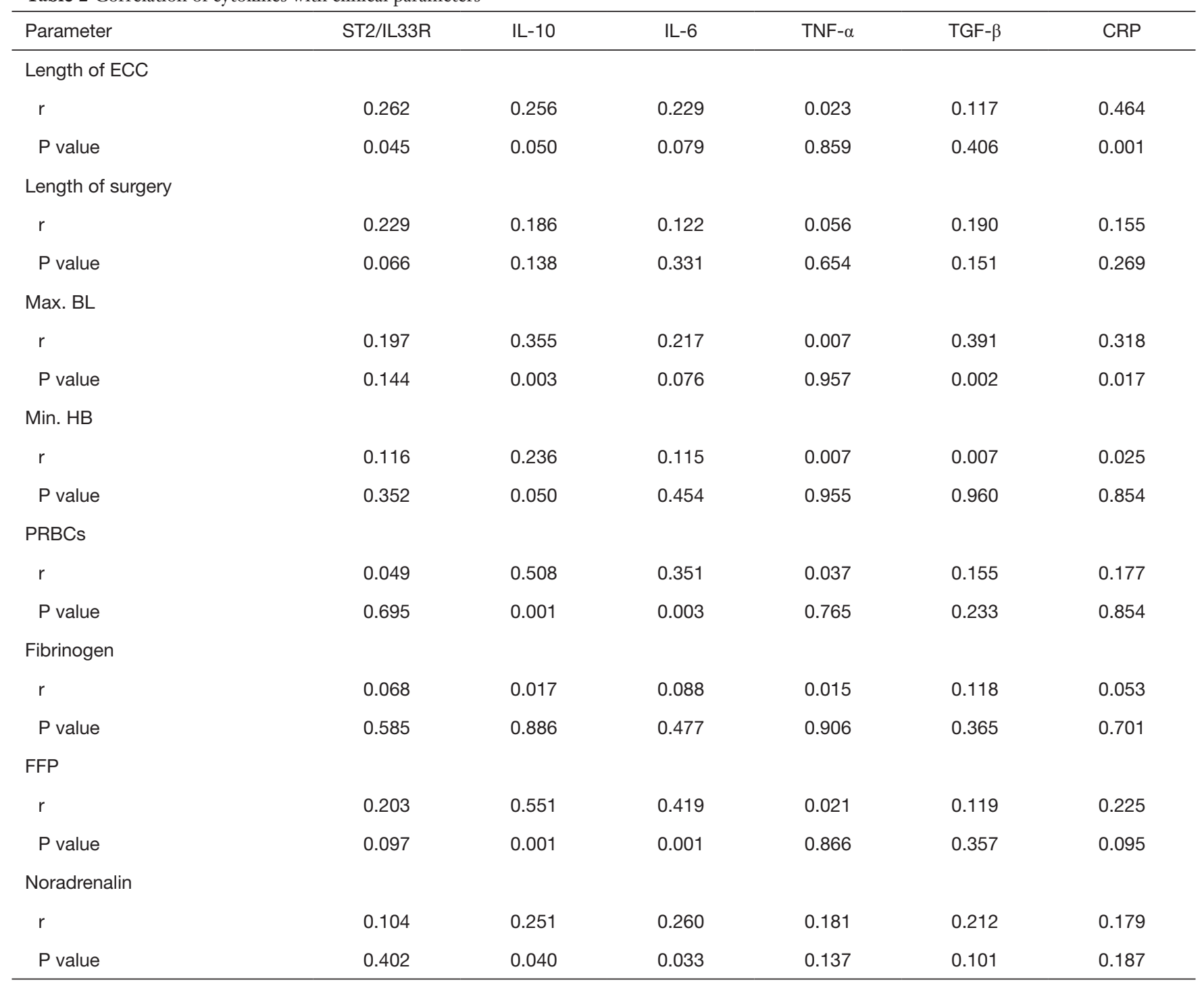

$\mathrm{BL}$, blood lactate concentration; CRP, C-reactive protein; ECC, extracorporeal circulation; ECMO, extracorporeal membrane oxygenation; FFP, fresh frozen plasma; IL, interleukin; Min. HB, minimum serum haemoglobin concentration; PRBCs, packed red blood cells; r, Pearson correlation coefficient; TGF, transforming growth factor; TNF, tumour necrosis factor

to re-transplantation for restrictive allograft syndrome (the initial LUTX was performed for end-stage IPF) that required postoperatively prolonged ECMO support for hemodynamic stability died on POD3 due to concomitant diffuse bleeding and massive central pulmonary embolism. Another patient who underwent LUTX for COPD with an early uneventful postoperative course (extubation POD1, transfer to the normal ward POD3) died on POD 10 due to acute bowel ischemia. One CTEPH patient with a high-risk hemodynamic profile died on POD5 because of persistent pulmonary hypertension after technically successful PEA with resultant prolonged v/a ECMO support.

\section{Discussion}

Indications for ECMO support have expanded rapidly beyond acute severe respiratory and cardiac failure and extracorporeal cardiopulmonary resuscitation; to semielective procedures, such as bridge to transplantation and elective cardiopulmonary support replacing $\mathrm{CPB}$ during 
Table 3 Applicability of cytokines to predict postoperative inflammation and organ dysfunction

\begin{tabular}{|c|c|c|c|c|c|}
\hline SOFA & OR & Sensitivity (\%) & Specificity (\%) & PPV (\%) & NPV (\%) \\
\hline IL-10 & 0.7 & 89 & 60 & 73 & 8 \\
\hline IL-6 & 18.6 & 97 & 80 & 82 & 30 \\
\hline TNF- $\alpha$ & 5.5 & 95 & 60 & 78 & 21 \\
\hline CRP & 2.4 & 95 & 80 & 38 & 11 \\
\hline
\end{tabular}

CRP, C-reactive protein; IL, interleukin; NPV, negative predictive value; OR, odds ratio; PPV, positive predictive value; SIRS, systemic inflammatory response syndrome; SOFA, sequential organ failure assessment score; TGF, transforming growth factor; TNF, tumour necrosis factor. Cut-offs were found using the Youden Index of absolute cytokine concentrations at end of surgery for SOFA: IL-10 $311 \mathrm{ng} / \mathrm{mL}$, IL-6 69.0 ng/mL, TNF- $\alpha 2.3$ ng/mL, ST2/IL33R 2,159 ng/mL, TGF- $\beta 17.2$ ng/mL and SIRS: ST2/IL33R 1,249 ng/mL, IL-10 82 ng/mL, IL-6 $19.0 \mathrm{ng} / \mathrm{mL}$, TNF- $\alpha 6.3 \mathrm{ng} / \mathrm{mL}$, TGF- $\beta 18,516 \mathrm{ng} / \mathrm{mL}$, CRP $0.84 \mathrm{mg} / \mathrm{dL}$, SOFA 8.5 .

surgery $(15,18,20)$. The main distinction between the acute, semi-elective and elective use of ECMO is the duration of support provided. Acute and semi-elective implanted ECMO can support patients with severe organ failure for weeks and months, whereas elective ECMO is similar to $\mathrm{CPB}$ only employed for hours. Semi- elective ECMO as a bridge to transplantation avoids mechanical ventilation and therefore reduces the risk of infection, functional impairment of other organs and muscle deconditioning $(18,21)$. Another important distinction between acute-, semi- and elective ECMO concerns mortality: while mortality rates under acute ECMO range from $37 \%$ to $76 \%$ depending on the indication, only $10 \%$ of all patients who were bridged to transplantation died in the last decade $(18,22)$. Until now, no intraoperative death related to elective ECMO support was reported (16).

In this study we revealed evidence for enhanced Th1 as well as Th2 responses at end of surgery in patients undergoing elective LUTX on ECMO and PEA on $\mathrm{CPB}$, which we did not observe in patients undergoing major pulmonary resections without ECC. The following observations point to an on/off phenomenon concerning SOFA and cytokine expression following major thoracic surgery on ECC support (CPB and ECMO): we did not observe perioperative differences in the quantitative and qualitative cytokine response or SOFA between PEA on CPB and LUTX on ECMO (stressing that no $t$-statistic was employed because of the inherent differences between patient groups and their respective surgery). Concerning the use of ECC the reasons for this on/off phenomenon may lie purely in the contact of blood components with tubing of ECC circuits. Other differences such as the use of an open (venous reservoir during $\mathrm{CPB}$ ) vs. closed circuit (ECMO), no suction of blood in the ECMO system, more bleeding because of full heparinization on $\mathrm{CPB}$, aortic cross clamping during $\mathrm{CPB}$, and others may be of subordinate significance for perioperative inflammation.

Few intraoperative methods were reported to reduce the incidence of inflammation. Beer et al. showed that continuous mechanical ventilation during $\mathrm{CABG}$ surgery reduced the systemic pro- and anti-inflammatory response (23). In our study, mechanical ventilation was continued during LUTX on ECMO and temporarily discontinued during deep hypothermia during PEA on $\mathrm{CPB}$. Furthermore, pulsatile flow during ECC attenuated the inflammatory response (24). CPB during PEA was performed with non-pulsatile perfusion; whereas various degrees of pulsatile flow were generated by the residual cardiac function of the patient during v/a ECMO, depending on the required respiratory and hemodynamic extracorporeal support (per protocol ECMO blood flow of $50 \%$ of the calculated cardiac output). Studies have shown that non-pulsatile perfusion causes a decrease in hemodynamic energy resulting in capillary collapse, microvascular shunting, and activation of inflammatory mediators $(25,26)$.

Our data did not allow differentiating between the effect of ECC and that of the surgical procedure itself as the use of ECMO during LUTX and CPB during PEA remains part of our standard surgical procedures. Since the institutional experience revealed improved short- and long-term outcomes with intraoperative ECMO-support a control group without ECMO could not be provided (18).

Propofol compared to sevoflurane anaesthesia can 
A

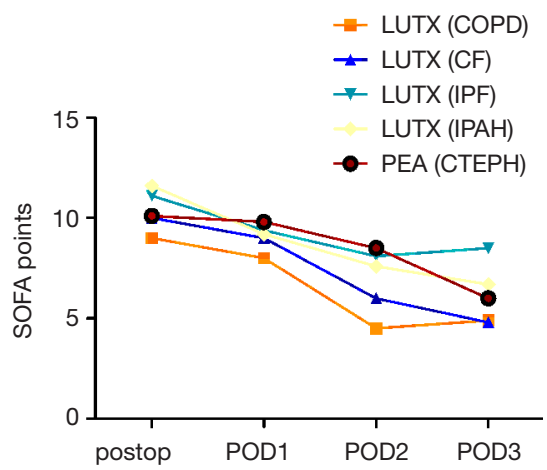

B

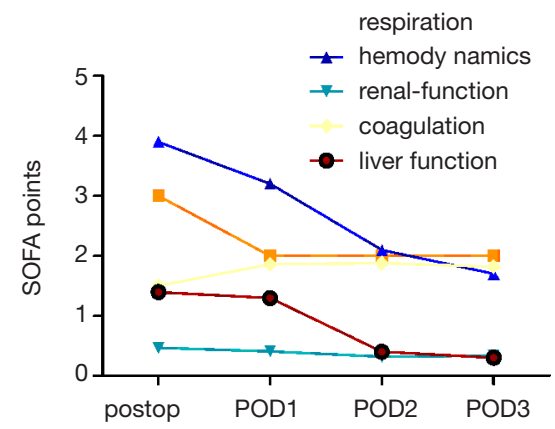

C

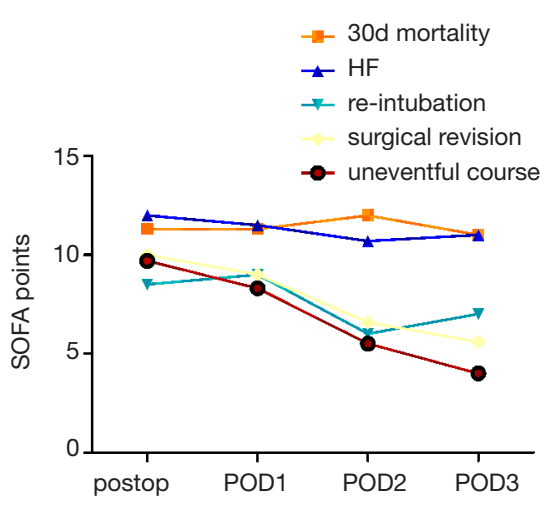

Figure 3 Relative changes in SOFA ( $\Delta$-SOFA) in the early postoperative period. A decrease from end of surgery to POD3 of $\triangle$-SOFA in patients admitted to ICU was depicted according to the underlying end stage pulmonary disease COPD, CF, IPF, IPAH and CTEPH patients (A). The postoperative course of the single factors of $\Delta$-SOFA are shown in (B). The postoperative $\triangle$-SOFA of 30-d mortality, patients requiring HF, patients who were reintubated, patients requiring surgical revision and patients with an uneventful postoperative course are shown in (C).

significantly reduce perioperative inflammation and protect pulmonary function $(27,28)$. Sevoflurane was employed in lung cancer (lung resection) and CTEPH (PEA) patients, due to its cardio-protective properties in patients with little or no ischemic heart disease (29). Patients with end- stage pulmonary disease undergoing LUTX were treated with intravenous perfusion of propofol for anaesthesia maintenance to avoid surgeon's exposure to volatile anaesthetic.

Large-scale studies validating the SOFA score in critically ill patients revealed increased mortality $(>50 \%)$ at a SOFA $\geq 10$ and $>95 \%$ at SOFA $>12(30,31)$. In our study only extremely high absolute SOFA $\geq 13$ at end of surgery showed poorer 30-d mortality in the Kaplan Mayer survival analysis. Since perioperative mortality in our ICU patient cohort with LUTX on ECMO and PEA on CPB patients was very low (3 out of 57 patients, $5 \%$ ), absolute SOFA scores may pertain to treatment strategies rather than real organ failure: the SOFA score assesses hepatic function only by determining total bilirubin levels. In a study on openheart surgery transient hyperbilirubinemia was evident by one third of all patients due to increased hemolysis caused by CPB (32). In our cohort continuous hemofiltration is frequently performed for excessive fluid overload removal in the presence of near normal renal function. In these patients, treatment strategies might change the renal SOFA score (creatinine levels and urine output) without genuine alterations in renal function. Further, CPB is known to lower platelet count in the early postoperative period, thus affecting the SOFA score by itself (33). In this study, we observed the same phenomena in patients undergoing LUTX on ECMO and PEA on CPB. Besides, SOFA has some limitation in actively sedated patients due to the use of the Glasgow coma scale to assess neurological function. Therefore, we customized the score by excluding the neurological assessment completely.

In patients undergoing cardiac surgery $\Delta$-SOFA varied among -3 to +1 according to length of ICU stay (33). In contrast, in our study $\triangle$-SOFA calculated from end of surgery to POD3 ranged from $-3.9 \pm 3.3$ to $-5.5 \pm 2.5$. The $\Delta$-SOFA was significantly smaller in patients requiring reintubation, hemofiltration or experiencing early mortality. Since SOFA is only applicable to ICU patients, the clinical course of patients transferred to the normal ward before POD 3 was not monitored by $\Delta$-SOFA. Therefore, $\Delta$-SOFA did not capture the patients with the best postoperative course and might underestimate the difference in $\Delta$-SOFA between patients with a complicated and an uncomplicated course. Patients undergoing lung resection were not admitted to ICU. Therefore, the qSOFA was used to detect organ failure (7). None of the patients after lung resection without ECC met the qSOFA criteria.

In our investigation, IL- 6 was the most sensitive 
parameter for detection of SOFA. IL-6 was reported to be a highly sensitive mediator of the acute phase reaction, allowing inflammatory conditions to be detected before the onset of associated clinical symptoms or before a rise in CRP (34). SOFA and cytokine release were phenomena irrespective of PGD after LUTX.

The chronological timeline of our measured cytokines IL-10, IL-6, ST2/IL33R and TNF- $\alpha$ were in line with previous findings of patients undergoing cardiac surgery $(35,36)$. Another previous study showed that IL-10 rose significantly in patients undergoing on-pump compared to off-pump CABG surgery (37). In our study, ECCtime correlated significantly with IL-10 and ST2/IL-33R concentrations, irrespective of total length of surgery. Further, serum concentrations of IL-10 prior to surgery were significantly increased in patients with semi-elective v/ v ECMO bridging compared to non-bridged patients.

Immunosuppression is an important part of ensuring allograft lung function after LUTX (38). Patients undergoing LUTX with elective ECMO received $1,000 \mathrm{mg}$ methylprednisolone 30 minutes prior to allograft reperfusion. After surgery three stages of immunosuppression followed: induction, maintenance, and treatment of acute rejection. Patients undergoing $\mathrm{PEA}$ received $100 \mathrm{mg}$ hydrocortisone prior to initiation of CPB in order to attenuate SIRS induced by CPB. Several trials investigated the use of steroids as a cheap method to mitigate the inflammatory response caused by CPB. The DECS trial included 4,494 patients requiring cardiac surgery with $\mathrm{CPB}$. Randomization of patients to intraoperative dexamethasone $1 \mathrm{mg} / \mathrm{kg}$ or placebo did not result in different outcomes. However, subgroup analysis focusing on patients with a EuroSCORE $>5$ demonstrated significant reductions in infection, delirium, and death under dexamethasone (39).

While immunosuppressive effects of PRBC transfusion were implied by reduced organ rejection after renal transplantation, subsequent studies observed proinflammatory effects and worse outcomes of LUTX in response to large-volume $\mathrm{PRBC}$ and platelet transfusion (40-42). In our study the number of transfused PRBC correlated positively with anti-inflammatory serum IL-10. Prophylactic FFP transfusion to critically ill non-bleeding patients resulted in decreased TNF- $\alpha$ levels (43). In our study, the amount of FFP transfusion correlated positively with IL-10 serum concentrations, maximal lactate levels and ECC-time.

Innate immune cells produce high amounts of lactate during inflammatory activation (44). In vitro treatment with lactate $>24$ h of peripheral blood mononuclear cells from healthy donors significantly modulated cytokine production with predominantly anti-inflammatory effects (45). We demonstrated a strong correlation between anti-inflammatory IL-10 and maximum lactate levels at end of surgery.

Our prospective, study has several limitations pertaining to its observational design. Hypothesis testing between the three patient groups: LUTX on ECMO, PEA on CPB and lung resection for cancer was not performed because of the inherent differences between the groups. Consequently, no statistical comparisons between the usage of ECMO and CPB can be drawn. The institutional LUTX experience clearly showed better outcomes with intraoperative ECMO support, since controlled reperfusion of a newly implanted pulmonary graft over a time period of 5 to 10 minutes saves the vulnerable organ. However, controlled reperfusion can only be achieved by using CPB or ECMO support. While, the potential beneficial effect of CPB during LUTX is hindered by an augmented intraoperative blood turnover and an increased risk of postoperative bleeding, ECMO support seems the best option to provide controlled reperfusion without increasing those risks $(46,47)$. Thus, no concurrent control groups of patients undergoing LUTX without intraoperative ECMO support could be provided $(16,18)$. Therefore, limitations concerning different surgical and anaesthesiological strategies for patients undergoing LUTX (ECMO), PEA (CPB) and lung resection (no ECC) are obvious and detailed in the manuscript. Moreover, patients undergoing LUTX received postoperative immunosuppression. However, specific immunosuppression pertaining to LUTX was launched after the second blood draw (at end of surgery). Therefore, only cytokine levels from POD1-POD5 were altered due to immunosuppression strategies in patients undergoing LUTX. Further, there is obvious unavoidable bias regarding the age and comorbidities of our cohort. Patients with COPD, IPF, CTEPH and lung cancer were by far older than CF and IPAH patients receiving bilateral LUTX. Patients with IPF are more likely to require ECMO bridging to transplant.

\section{Conclusions}

Elective thoracic surgery on ECC support followed by an uneventful postoperative course and excellent outcomes triggered an immediate rise and concomitant fall of inflammation as observed in serum cytokine release and SOFA criteria. High absolute SOFA scores in the presence 


\section{Page 14 of 16}

of an uncomplicated postoperative course may pertain to specific management strategies rather than organ failure. IL-6 serum concentrations, extremely high SOFA $\geq 13$ and a missing decline in $\Delta$-SOFA may predict outcomes. Future studies investigating the potential biological significance of perioperative cytokine release are warranted.

\section{Acknowledgments}

We acknowledge all participating colleagues to share their knowledge. We are grateful for their efforts and the time they have spent supporting the study. We thank the research laboratories ARGE Moser and ARGE Ankersmit (FOLAB Chirurgie) of the department of surgery, Medical University Vienna for funding

Funding: This work was supported by the institutional research laboratories ARGE Moser (FOLAB Chirurige) and FFG-Grant “APOSEC” (\#852748; 2015-2018).

\section{Footnote}

Reporting Checklist: The authors have completed the STROBE reporting checklist. Available at http://dx.doi. org/10.21037/atm-20-4771

Data Sharing Statement: Available at http://dx.doi. org/10.21037/atm-20-4771

Peer Review File: Available at http://dx.doi.org/10.21037/ atm-20-4771

Conflicts of Interest: All authors have completed the ICMJE uniform disclosure form (available at http://dx.doi. org/10.21037/atm-20-4771). The authors have no conflicts of interest to declare.

Ethical Statement: The authors are accountable for all aspects of the work in ensuring that questions related to the accuracy or integrity of any part of the work are appropriately investigated and resolved. The study was conducted in accordance with the Declaration of Helsinki (as revised in 2013). The study was approved by the Institutional Ethics Committee of the Medical University of Vienna (EK1363/2018). Written informed consent was obtained from all study participants.

Open Access Statement: This is an Open Access article distributed in accordance with the Creative Commons
Veraar et al. Transient perioperative inflammation following LUTX

Attribution-NonCommercial-NoDerivs 4.0 International License (CC BY-NC-ND 4.0), which permits the noncommercial replication and distribution of the article with the strict proviso that no changes or edits are made and the original work is properly cited (including links to both the formal publication through the relevant DOI and the license). See: https://creativecommons.org/licenses/by-nc-nd/4.0/.

\section{References}

1. Millar JE, Fanning JP, McDonald CI, et al. The inflammatory response to extracorporeal membrane oxygenation (ECMO): a review of the pathophysiology. Crit Care 2016;20:387.

2. Taghavi S, Ankersmit HJ, Wieselthaler G, et al. Extracorporeal membrane oxygenation for graft failure after heart transplantation: recent Vienna experience. J Thorac Cardiovasc Surg 2001;122:819-20.

3. Lang G, Taghavi S, Aigner C, et al. Extracorporeal membrane oxygenation support for resection of locally advanced thoracic tumors. Ann Thorac Surg 2011;92:264-70.

4. Ko WJ, Chen YS, Chou NK, et al. ECMO support for single lung transplantation. Transplant Proc 2001;33:1939-41.

5. Pereszlenyi A, Lang G, Steltzer H, et al. Bilateral lung transplantation with intra- and postoperatively prolonged ECMO support in patients with pulmonary hypertension. Eur J Cardiothorac Surg 2002;21:858-63.

6. Maurer JR, Frost AE, Estenne M, et al. International guidelines for the selection of lung transplant candidates. The International Society for Heart and Lung Transplantation, the American Thoracic Society, the American Society of Transplant Physicians, the European Respiratory Society. Heart Lung 1998;27:223-9.

7. Raith EP, Udy AA, Bailey M, et al. Prognostic Accuracy of the SOFA Score, SIRS Criteria, and qSOFA Score for In-Hospital Mortality Among Adults With Suspected Infection Admitted to the Intensive Care Unit. JAMA 2017;317:290-300.

8. Vincent JL, Moreno R, Takala J, et al. The SOFA (Sepsisrelated Organ Failure Assessment) score to describe organ dysfunction/failure. On behalf of the Working Group on Sepsis-Related Problems of the European Society of Intensive Care Medicine. Intensive Care Med 1996;22:707-10.

9. Mildner RJ, Taub N, Vyas JR, et al. Cytokine imbalance in infants receiving extracorporeal membrane oxygenation 
for respiratory failure. Biol Neonate 2005;88:321-7.

10. Boyle EM, Jr., Pohlman TH, Johnson MC, et al. Endothelial cell injury in cardiovascular surgery: the systemic inflammatory response. Ann Thorac Surg 1997;63:277-84.

11. Mojcik CF, Levy JH. Aprotinin and the systemic inflammatory response after cardiopulmonary bypass. Ann Thorac Surg 2001;71:745-54.

12. Abrams D, Combes A, Brodie D. Extracorporeal membrane oxygenation in cardiopulmonary disease in adults. J Am Coll Cardiol 2014;63:2769-78.

13. Mc IRB, Timpa JG, Kurundkar AR, et al. Plasma concentrations of inflammatory cytokines rise rapidly during ECMO-related SIRS due to the release of preformed stores in the intestine. Lab Invest 2010;90:128-39.

14. Chen Q, Yu W, Shi J, et al. The effect of venovenous extra-corporeal membrane oxygenation (ECMO) therapy on immune inflammatory response of cerebral tissues in porcine model. J Cardiothorac Surg 2013;8:186.

15. Mosier JM, Kelsey M, Raz Y, et al. Extracorporeal membrane oxygenation (ECMO) for critically ill adults in the emergency department: history, current applications, and future directions. Crit Care 2015;19:431.

16. Moser B, Jaksch P, Taghavi S, et al. Lung transplantation for idiopathic pulmonary arterial hypertension on intraoperative and postoperatively prolonged extracorporeal membrane oxygenation provides optimally controlled reperfusion and excellent outcome. Eur J Cardiothorac Surg 2018;53:178-85.

17. Jamieson SW, Kapelanski DP, Sakakibara N, et al. Pulmonary endarterectomy: experience and lessons learned in 1,500 cases. Ann Thorac Surg 2003;76:1457-62; discussion 1462-4.

18. Benazzo A, Schwarz S, Frommlet F, et al. Twenty-year experience with extracorporeal life support as bridge to lung transplantation. J Thorac Cardiovasc Surg 2019;157:2515-25.e10.

19. Bernardi MH, Rinoesl H, Dragosits K, et al. Effect of hemoadsorption during cardiopulmonary bypass surgery a blinded, randomized, controlled pilot study using a novel adsorbent. Crit Care 2016;20:96.

20. Shekar K, Mullany DV, Thomson B, et al. Extracorporeal life support devices and strategies for management of acute cardiorespiratory failure in adult patients: a comprehensive review. Crit Care 2014;18:219.

21. O'Brien G, Criner GJ. Mechanical ventilation as a bridge to lung transplantation. J Heart Lung Transplant 1999; 18:255-65.
22. Aubron C, Cheng AC, Pilcher D, et al. Factors associated with outcomes of patients on extracorporeal membrane oxygenation support: a 5-year cohort study. Crit Care 2013;17:R73.

23. Beer L, Szerafin T, Mitterbauer A, et al. Ventilation during cardiopulmonary bypass: impact on heat shock protein release. J Cardiovasc Surg (Torino) 2014;55:849-56.

24. Schmitz KJ, Unkel C, Grabellus F, et al. Melanotic schwannoma of the neck mimicking a malignant melanoma. Eur Arch Otorhinolaryngol 2005;262:182-5.

25. Undar A, Ji B, Lukic B, et al. Quantification of perfusion modes in terms of surplus hemodynamic energy levels in a simulated pediatric CPB model. ASAIO J 2006;52:712-7.

26. Orime Y, Shiono M, Hata H, et al. Cytokine and endothelial damage in pulsatile and nonpulsatile cardiopulmonary bypass. Artif Organs 1999;23:508-12 .

27. Tian HT, Duan XH, Yang YF, et al. Effects of propofol or sevoflurane anesthesia on the perioperative inflammatory response, pulmonary function and cognitive function in patients receiving lung cancer resection. Eur Rev Med Pharmacol Sci 2017;21:5515-22.

28. Schneemilch CE, Bank U. Release of pro- and antiinflammatory cytokines during different anesthesia procedures. Anaesthesiol Reanim 2001;26:4-10.

29. Jakobsen CJ, Berg H, Hindsholm KB, et al. The influence of propofol versus sevoflurane anesthesia on outcome in 10,535 cardiac surgical procedures. J Cardiothorac Vasc Anesth 2007;21:664-71.

30. Vincent JL, de Mendonca A, Cantraine F, et al. Use of the SOFA score to assess the incidence of organ dysfunction/failure in intensive care units: Results of a multicenter, prospective study. Critical Care Medicine 1998;26:1793-800.

31. Ferreira FL, Bota DP, Bross A, et al. Serial evaluation of the SOFA score to predict outcome in critically ill patients. JAMA 2001;286:1754-8.

32. Wang MJ, Chao A, Huang CH, et al. Hyperbilirubinemia after cardiac operation. Incidence, risk factors, and clinical significance. J Thorac Cardiovasc Surg 1994;108:429-36.

33. Patila T, Kukkonen S, Vento A, et al. Relation of the Sequential Organ Failure Assessment score to morbidity and mortality after cardiac surgery. Ann Thorac Surg 2006;82:2072-8.

34. Fink-Neuboeck N, Lindenmann J, Bajric S, et al. Clinical impact of interleukin 6 as a predictive biomarker in the early diagnosis of postoperative systemic inflammatory response syndrome after major thoracic surgery: A prospective clinical trial. Surgery 2016;160:443-53. 


\section{Page 16 of 16}

35. Szerafin T, Niederpold T, Mangold A, et al. Secretion of soluble ST2 - possible explanation for systemic immunosuppression after heart surgery. Thorac Cardiovasc Surg 2009;57:25-9.

36. Beer L, Szerafin T, Mitterbauer A, et al. Continued mechanical ventilation during coronary artery bypass graft operation attenuates the systemic immune response. Eur J Cardiothorac Surg 2013;44:282-7.

37. Szerafin T, Brunner M, Horvath A, et al. Soluble ST2 protein in cardiac surgery: a possible negative feedback loop to prevent uncontrolled inflammatory reactions. Clin Lab 2005;51:657-63.

38. Thompson ML, Flynn JD, Clifford TM. Pharmacotherapy of lung transplantation: an overview. J Pharm Pract 2013;26:5-13.

39. Dieleman JM, Nierich AP, Rosseel PM, et al. Intraoperative high-dose dexamethasone for cardiac surgery: a randomized controlled trial. JAMA 2012;308:1761-7.

40. Shaz BH, Stowell SR, Hillyer CD. Transfusion-related acute lung injury: from bedside to bench and back. Blood 2011;117:1463-71.

41. Bilgin YM, van de Watering LMG, Versteegh MIM, et al. Effects of allogeneic leukocytes in blood transfusions during cardiac surgery on inflammatory mediators and postoperative complications. Crit Care Med

Cite this article as: Veraar C, Schwarz S, Thanner J, Direder M, Boehm PM, Harnoncourt L, Ortmayr J, Veraar C, Mascherbauer J, Klepetko W, Dworschak M, Ankersmit HJ, Moser B. Transient perioperative inflammation following lung transplantation and major thoracic surgery with elective extracorporeal support: a prospective observational study. Ann Transl Med 2021;9(5):385. doi: 10.21037/atm-20-4771
Veraar et al. Transient perioperative inflammation following LUTX

2010;38:546-52.

42. Pena JJ, Bottiger BA, Miltiades AN. Perioperative Management of Bleeding and Transfusion for Lung Transplantation. Semin Cardiothorac Vasc Anesth 2020;24:74-83.

43. Straat M, Muller MC, Meijers JC, et al. Effect of transfusion of fresh frozen plasma on parameters of endothelial condition and inflammatory status in nonbleeding critically ill patients: a prospective substudy of a randomized trial. Crit Care 2015;19:163.

44. Palsson-McDermott EM, O'Neill LA. The Warburg effect then and now: from cancer to inflammatory diseases. Bioessays 2013;35:965-73.

45. Ratter JM, Rooijackers HMM, Hooiveld GJ, et al. In vitro and in vivo Effects of Lactate on Metabolism and Cytokine Production of Human Primary PBMCs and Monocytes. Front Immunol 2018;9:2564.

46. Aigner C, Wisser W, Taghavi S, et al. Institutional experience with extracorporeal membrane oxygenation in lung transplantation. Eur J Cardiothorac Surg 2007;31:468-73; discussion 473-4.

47. Hoetzenecker K, Benazzo A, Stork T, et al. Bilateral lung transplantation on intraoperative extracorporeal membrane oxygenator: An observational study. J Thorac Cardiovasc Surg 2020;160:320-7.e1. 